\title{
Uma coleção herborizada PANC como recurso didático para o ensino de biologia
}

\author{
An UFP herborized collection as a didactic resource for teaching biology
}

\author{
Alaine Santana de Farias ${ }^{\oplus} \bowtie$ \& Marla Ibrahim Uehbe de Oliveira ${ }^{1}{ }^{(1)}$
}

\footnotetext{
1. Departamento de Biologia, Universidade Federal de Sergipe, São Cristóvão, Sergipe, Brasil
}

\section{Palavras-chave \\ Botânica. Ciências. Coleção biológica. Herbários. Plantas alimentícias não convencionais.}

\section{Keywords \\ Botany. Science. Biological collection. Her- baria. Unconventional food plants.}

\section{Doi}

doi.org/10.33447/paubrasilia.v3i2.39

Recebido em: 26/05/2020

Aceite em: 31/07/2020

Editor responsável: Gleidson V.

Marques (UFSB)

ISSN: $2595-6752$

\begin{abstract}
Resumo
No ensino de biologia ainda persistem a memorização excessiva de conceitos e teorias, além da descontextualização. As coleções biológicas, quando relacionadas às Plantas Alimentícias Não Convencionais (PANC), podem ser uma excelente forma de conectar o conteúdo botânico com plantas presentes no dia a dia dos alunos. Assim, este trabalho teve como objetivo relatar a elaboração de uma coleção didática herborizada de PANC para ser utilizada por professores em aulas de biologia, visando a contribuir para uma aprendizagem mais atrativa e significativa. Foram pesquisadas informações sobre as PANC e selecionadas espécies presentes no estado de Sergipe que poderiam contribuir com o processo de ensino e aprendizagem. Em meio à baixa motivação presente na educação atual, é necessário buscar alternativas para melhorar a qualidade do ensino e, consequentemente, formar cidadãos cientes do mundo que está a sua volta.
\end{abstract}

\begin{abstract}
Excessive memorization of concepts and theories persists in classes of Science and Biology, besides the decontextualization. Biological collections, when related to Unconventional Food Plants (UFP), can be an excellent way to connect botanical content with plants present in student's regular lives. Thus, this work aimed to report the elaboration of a dried didactic collection of UFP to be used by teachers in Biology classes to contribute to a more attractive and meaningful learning. Information about UFP was searched and species in the state of Sergipe that could contribute to the teaching and learning process were selected. Although low motivation is still present in current education, it is necessary to look for alternatives to improve the quality of education and, consequently, to raise citizens aware of the world around them.
\end{abstract}

\section{Introdução}

A educação é tida como processo de grande importância para a promoção de mudança social, acompanhando o indivíduo durante toda sua vida. Esta envolve principalmente a transmissão de conhecimento através do ensino, que é visto como enfoque prático e formal da educação. Ele dispõe de alicerces e técnicas específicas que promovem o desenvolvimento e a evolução da sociedade (Carvalho, 2000).

O ensino nas escolas brasileiras utiliza predominantemente a abordagem tradicional, que apresenta como principal característica a centralização no professor. Ele retém a autoridade de decisão em qualquer situação e assegura a transmissão de informações aos educandos. Nessa abordagem, o aluno atua de forma passiva, como depositário de conhecimentos definidos como importantes. O ensino se resume ao repasse de informações, que seguem padrões sistematizados. Assim, a inteligência é definida como a capacidade de armazenar esse conhecimento (Mizukami, 1986; Carvalho, 2000). 
A abordagem tradicional de ensino, embora ainda eficaz, não é mais suficiente para a aprendizagem das gerações atuais. Com os avanços na tecnologia, os jovens possuem cada vez mais um ritmo acelerado, e o tempo de uma aula, no qual o aluno apenas ouve o professor, pode parecer monótono. Esta desmotivação para acompanhar as aulas tradicionais pode resultar em dificuldades no processo de ensino e aprendizagem (Fagundes; Gonzalez, 2006; Cordeiro, 2013).

Segundo Freire (2013), o professor pode estimular a curiosidade do aluno, conectando a realidade vivida por ele e o conteúdo visto em sala de aula. Isto possibilitaria a construção do conhecimento e a autonomia desse aluno, tornando-o agente ativo. Sendo assim, é preciso analisar os conteúdos ensinados, a forma de ensino e o que se deseja como educador (Fagundes; Gonzalez, 2006).

No ensino de ciências e biologia, são indicadas como limitações para a aprendizagem a memorização excessiva de conceitos e teorias, descontextualização, falta de interdisciplinaridade e predomínio do método expositivo (Carvalho, 2000; Santori; Santos, 2015; Krasilchik, 2016). Krasilchik (2016) aponta a comunicação oral como um dos principais obstáculos, que pode levar os alunos a considerar a disciplina entediante, sem interação entre professor e aluno.

No ensino da botânica, área do conhecimento que estuda plantas, algas e fungos (Esteves, 2011), podem ser observados fatores que dificultam o aprendizado: falta de material didático, de atividades práticas, e de contextualização da teoria com a realidade. A constante presença de termos técnicos é outro agravante, pois os mesmos não costumam fazer parte do cotidiano dos alunos (Menezes et al., 2008).

Nas Orientações Curriculares Nacionais para o Ensino Médio (Brasil, 2006), a botânica tem o compromisso de contribuir para que os educandos desenvolvam habilidades necessárias para a compreensão do papel do homem na natureza. $\mathrm{O}$ ensino dentro dessa área de conhecimento exige criatividade e dedicação, fazendo-se necessário transformar a informação em conhecimentos para os alunos. O professor, como agente construtor de pessoas capacitadas e críticas, precisa repensar suas metodologias e práticas de ensino (Silva, 2015).

A Base Nacional Comum Curricular (BNCC) da área de ciências da natureza e suas tecnologias, na definição das competências e habilidades da área, cita a necessidade da contextualização social, cultural e histórica dos conhecimentos científicos, trazendo-os para o cotidiano dos estudantes, estimulando o protagonismo desses jovens no enfrentamento de questões sociais (Brasil, 2018).

Deste modo, as coleções didáticas biológicas, além de proporcionarem o conhecimento da biodiversidade, possibilitam estudos relacionados a áreas como morfologia, taxonomia, evolução, entre outras, propiciando a expansão dos conhecimentos referentes às espécies colecionadas. Também, podem desenvolver interesses nos alunos, como por exemplo, a vontade de interagir com a natureza, buscar conservar e realizar coletas (Santori; Santos, 2015).

Segundo Marandino et al. (2009), o ensino de ciências e biologia é historicamente apontado pelo uso de objetos com funções diversas no contexto escolar como demonstrar, ilustrar, explicar. Desta maneira, fazer o uso de coleções biológicas é uma forma de manter presente essa tradição tão importante, que torna as aulas mais atrativas e participativas. Entre as coleções biológicas há o herbário, e dentre as coleções auxiliares, há a coleção didática.
O herbário representa uma coleção de plantas, algas e fungos preservados, que têm como finalidade a documentação da diversidade da flora. As informações nele contidas facilitam a identificação de novas espécies através da comparação com outras já identificadas. Além disso, fornecem dados para estudos e pesquisas, possibilitando a análise da diversidade do passado e presente. Assim, contribuem para a preservação e formação cidadã, com a divulgação dos conhecimentos através de exposições, visitas guiadas, palestras (Esteves, 2011; Maia et al., 2017).

A flora brasileira possui uma grande diversidade, composta por cerca de 46.600 espécies (Flora do Brasil 2020 em construção, 2020). Muitas delas, que são comumente denominadas de invasoras, daninhas, pragas ou mato, possuem potencial alimentício ainda desconhecido pela maior parte da população. Por esta razão, são chamadas de Plantas Alimentícias Não Convencionais ou PANC (Kinupp; Barros, 2004; Kinupp; Lorenzi, 2017).

A diversidade de plantas alimentícias é grandiosa, mas $90 \% \mathrm{da}$ alimentação mundial se resumem a apenas 20 espécies. Só no Brasil, estima-se a existência de 2.000 a 3.000 espécies com potencial alimentício, a maioria de angiospermas (Kinupp; Barros, 2004; Kinupp; Lorenzi, 2017). Em estudo realizado por Barreira et al. (2015), foram encontradas PANC em pastos, em meio a cultivos agrícolas, hortas e segmentos florestais, a maioria apresentando ciclos reprodutivos curtos, propagando-se facilmente. No entanto, esta capacidade é pouco explorada na agricultura nacional, que optou por uma especialização de culturas (Kinupp, 2009; Kinupp; Lorenzi, 2017).

Além de contribuir com a valorização da biodiversidade e diversificação alimentar, o consumo das PANC colabora para uma agricultura conservacionista. Isto se deve ao fato de serem espécies adaptadas ao meio onde ocorrem, não havendo necessidade de uso de agrotóxicos, que são prejudiciais à saúde humana e ao meio ambiente (Kinupp; Barros, 2004).

Barreira et al. (2015) obtiveram resultados que apontam que gerações mais novas possuem menos conhecimentos sobre as PANC. Isto pode ter acontecido em razão da saída dos jovens da zona rural para a urbana, por motivos como trabalho e estudos. Sendo assim, não houve convivência com os mais velhos, que possuem os conhecimentos culturais sobre as PANC. Kinupp e Barros (2004) indicam ainda como problemas a falta de informações sobre o consumo dessas plantas e seu modo de preparo, além da resistência por serem consideradas daninhas.

Considerando-se todas estas informações, este trabalho teve como objetivo relatar a elaboração de uma coleção didática herborizada de Plantas Alimentícias Não Convencionais (PANC) para ser utilizada por professores em aulas de biologia, contribuindo para uma aprendizagem mais atrativa e significativa. A escolha do tema deveu-se à necessidade sentida de disseminar conhecimentos sobre o mesmo, por ser considerado de relevância social e, consequentemente, interessante para ser abordado na sala de aula. Seu desenvolvimento aconteceu de outubro de 2018 a fevereiro de 2019, dividido em duas fases: montagem da coleção botânica didática de PANC e construção de um manual para o professor. 


\section{Montagem da Coleção Herborizada Didática de PANC}

\section{Escolha das PANC e coleta de material}

A escolha das espécies a serem trabalhadas na montagem da coleção didática foi feita a partir de informações contidas em referências bibliográficas especializadas em PANC, especialmente Kinupp e Lorenzi (2017). Em seguida, foi feita verificação da ocorrência das mesmas no estado de Sergipe através dos registros no Herbário da Universidade Federal de Sergipe (ASE) e de dados nos sítios eletrônicos speciesLink (Centro de Referência em Informação Ambiental, 2019) e Flora do Brasil 2020 (Flora do Brasil 2020 em construção, 2020).

Após esta etapa, onze espécies foram selecionadas com base na facilidade de acesso para coleta e conexão entre a possível realidade do aluno e o conteúdo ensinado. As coletas aconteceram em três municípios de Sergipe - Ribeirópolis, São Cristóvão e São Miguel do Aleixo -, e os exemplares foram herborizados seguindo as técnicas usuais propostas por Peixoto e Maia (2013). Frutos foram preservados em potes contendo álcool $70 \%$ para uma observação das estruturas de forma íntegra. A identificação foi confirmada por comparação com materiais depositados nos herbários através do sítio eletrônico speciesLink (Centro de Referência em Informação Ambiental, 2019), e seguiu a classificação do Angiosperm Phylogeny Group - APG IV (APG, 2016). A montagem das amostras foi feita em cartolina branca, contando com informações de coleta em uma etiqueta de dados.

\section{Manual para o professor}

O manual foi elaborado pelas autoras, fundamentado em referências bibliográficas especializadas - como Prata et. al (2013), Santos (2013) e Kinupp e Lorenzi (2017) -, impresso em papel A4 e encadernado para garantir durabilidade. Ele foi pensado para orientar o professor quanto ao uso da coleção herborizada didática de PANC, além da diversidade e importância dessas plantas. Foram explicados os procedimentos necessários para realizar a coleta e montagem da coleção botânica, para o incremento da coleção, a reposição de amostras danificadas ou a construção de uma nova coleção, caso o professor deseje.

Fotos representativas e explicativas foram anexadas para auxiliar a compreensão da montagem da coleção. Foram indicadas possibilidades para a utilização do recurso durante as aulas e sugeridas leituras complementares para auxiliar a identificação das plantas, locais para coletas (como jardins e quintais), e os cuidados a serem considerados. O manual encontra-se disponível no repositório institucional da Universidade Federal de Sergipe (UFS), e há o interesse em transformá-lo em e-book para aplicação futura em proposta didática.

\section{Discussão Sobre a Elaboração do Material Didático}

Coleções botânicas são exploradas como materiais didáticos por diversos autores, como Bessa (2011), Fagundes e Gonzalez (2006), e Santos (2013). No entanto, a coleção aqui apresentada difere-se por explorar pela primeira vez em Sergipe as PANC, conhecidas por se tratar de espécies que normalmente apresentam ampla distribuição geográfica e potencial econômico e ecológico não reconhecido pela maior parte da população.

As plantas utilizadas para elaboração da coleção foram: Nopalea cochenillifera (L.) Salm-Dyck (Cactaceae) (Figura 1a), Byrsonima crassifolia (L.) Kunth (Malpighiaceae), Amaranthus deflexus L. (Amaranthaceae), Portulaca oleracea L. (Portulacaceae), Turnera subulata Sm. (Passifloraceae) (Figura 1b), Caesalpinia pulcherrima (L.) Sw. (Fabaceae) (Figura 1c), Pachira aquatica Aubl. (Malvaceae) (Figura 1d), Solanum americanum Mill. (Solanaceae), Licania tomentosa Benth. (Chrysobalanaceae), Talinum paniculatum (Jacq.) Gaertn. (Talinaceae) e Morinda citrifolia L. (Rubiaceae).

Estas espécies são nativas do país - exceto Nopalea cochenillifera, incluída no trabalho em razão de seu uso em culturas no nordeste brasileiro -, ornamentais ou consideradas invasoras, compondo a arborização urbana e jardins, encontradas em canteiros, praças e quintais. Destacar essas espécies na sala de aula reforça o conhecimento da flora local por parte dos alunos. Estudos demonstraram que estudantes em geral não possuem uma percepção da identidade da flora regional (Silva, 2011). Assim, é preciso incluir assuntos transversais relacionados à Botânica nas escolas, principalmente em escolas urbanas, nas quais os alunos possuem menos contato com a flora. Essa integração, além de apresentar a flora da região, possibilita a utilização de conhecimentos adquiridos no ambiente escolar e em seu cotidiano (Gamarra-Rojas et al., 2003).

Figura 1. Plantas Alimentícias Não Convencionais utilizadas neste estudo. a. Nopalea cochenillifera (L.) Salm-Dyck. b. Turnera subulata Sm. c. Caesalpinia pulcherrima (L) Sw. d. Pachira aquatica Aubl. Fonte: Autoras.
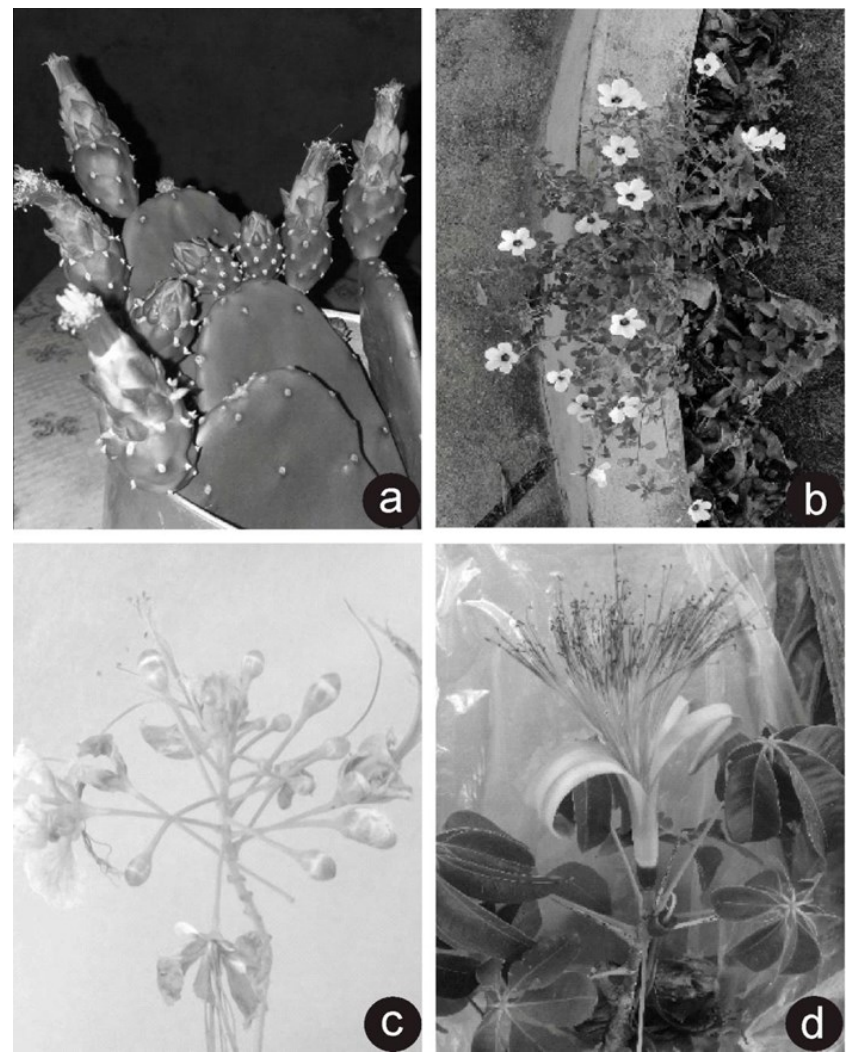

Importante destacar o trabalho de Marandino et al. (2009), que produz uma reflexão a respeito da utilização de coleções biológicas no processo de aprendizagem nas escolas e em espaços não 
formais de ensino, mostrando o auxílio no ensino e aprendizagem das disciplinas escolares. De acordo com a Base Nacional Comum Curricular (BNCC), é preciso propiciar experiências que promovam as aprendizagens essenciais para o enfrentamento dos desafios atuais da sociedade (Brasil, 2018).

Para tanto, o manual denominado "Manual PANC: conceitos, dicas de uso e curiosidades" (Figura 2) foi elaborado e dividido nas sessões: (i) Conhecendo as PANC e a Flora de Sergipe; (ii) Coleções biológicas: herbário didático; (iii) Coleção herborizada de PANC; (iv) Quando e como utilizar a coleção herborizada PANC; (v) Como montar essa coleção?; (vi) Sugestão de material complementar.

Estas sessões do manual foram definidas por serem consideradas relevantes para o professor, pois se acredita que trazem de forma resumida e simplificada informações sobre a construção da coleção didática e sua utilização. Estas mesmas atividades foram realizadas por Ferreira e Libano (2011), que mencionaram a importância da confecção de um manual de uso do recurso didático para o professor por facilitar a aplicação do material, possibilitando o alcance dos objetivos estabelecidos. Para professores de biologia, é indispensável que atividades como a coleção didática venham acompanhadas de um roteiro, pois o mesmo é de simples adaptação e claro, indicando materiais acessíveis, diferente do que ocorre em manuais para coleções científicas (Ota, 2012; Santos, 2013).

A sessão sobre as PANC e a flora de Sergipe traz para o profes-

Figura 2. Representação do manual para professores. a. Capa. b. Sumário com informações do conteúdo abordado. Fonte: Autoras.
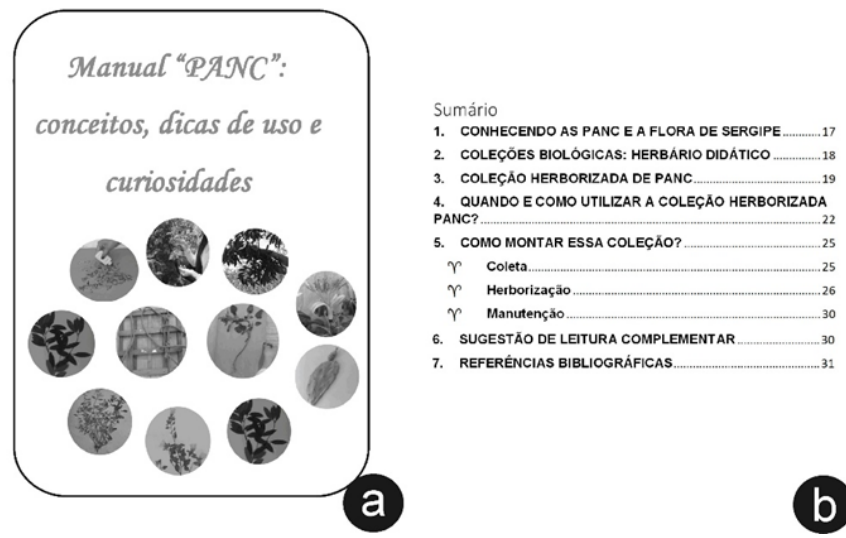

sor uma síntese sobre as mesmas, para serem apresentadas aos alunos ao trabalhar a coleção. É importante falar sobre as PANC, já que estas espécies alimentícias estão presentes em nosso cotidiano, fazendo parte da diversidade, e cuja importância comumente não é conhecida (Kinupp; Lorenzi, 2017). Elas são uma excelente forma de contextualizar o conteúdo botânico e assim facilitar o processo de aprendizagem. Essa contextualização é necessária porque os alunos possuem dificuldade para abstrair conceitos e termos, sendo preciso vivenciar a ciência no dia a dia (Ota, 2012; Acordi; Pasa, 2014).

As sessões sobre herbário didático (Figura 3) e o passo a passo para montar o mesmo apresentam as definições e características de herbários e o roteiro para montagem, além da sua importância para o processo pedagógico. A coleção botânica é um recurso eficiente para o ensino, mostrando resultados satisfatórios e motivadores para os alunos (Fagundes; Gonzalez, 2006; Bessa, 2011; Santos, 2013).
O uso de coleções didáticas auxilia a diversificar o ensino, porque possibilita a aplicação de atividades diversas, como observação e comparação de estruturas, relação entre morfologia e adaptação ao ambiente (Santos, 2013). Em pesquisa realizada por Bessa (2011), os alunos demonstraram interesse pela coleção, fazendo perguntas, participando ativamente das aulas, mencionando que o material ajudou na compreensão do conteúdo. O professor da turma também aprovou o material, afirmando a inclusão deste nas suas aulas. Resultados semelhantes foram obtidos por Fagundes e Gonzalez (2006), nos quais os alunos informaram que o uso da coleção herborizada facilitou o aprendizado dos termos técnicos, porque esta permitiu o envolvimento de forma participativa com os conteúdos.

Figura 3. Informações contidas no manual para professores. a. Processos envolvidos na herborização de plantas. b. Anotações sobre a coleta e amostra finalizada com capa protetora. Fonte: Autoras.
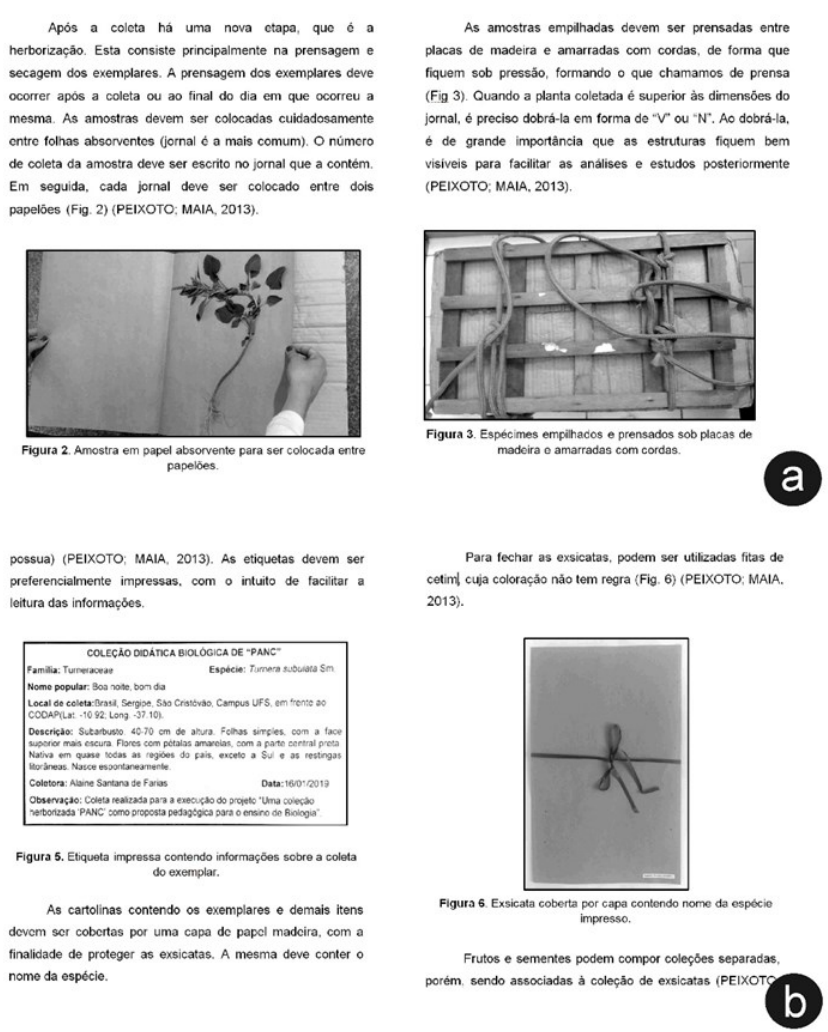

A coleção herborizada de PANC é tratada em uma sessão com o intuito de apresentar a sua importância. A mesma constitui um significativo recurso didático, haja vista que não foram encontrados outros trabalhos abordando-a em sala de aula. A coleção aqui discutida pode contribuir não somente para o ensino de botânica, mas de outras áreas da biologia como alimentação e evolução, por exemplo.

Nesta sessão, há um resumo das características de cada espécie presente na coleção. São informadas características morfológicas, origem, distribuição, nome popular, partes comestíveis, maneira de consumir. Sobre este último tópico, informações sobre as estruturas com potencial comestível variaram de acordo com cada espécie. Por exemplo, algumas delas possuem as folhas comestíveis, como o caruru ou bredo (Amaranthus deflexus), a beldroega (Portulaca oleracea) e o major gomes (Talinum paniculatum). Outras possuem flores comestíveis, como a boa-noite (Turnera subulata) e o flamboian-de-jardim (Caesalpinia pulcherrima). Além disso, podem ser utilizadas no preparo de sucos, refogados, saladas, sopas (Kinupp; Lorenzi, 2017). 
Através das dicas de uso para a coleção herborizada de PANC, o professor terá várias possibilidades para utilizar a mesma, podendo fazê-la em diversos momentos, como aulas de anatomia e morfologia vegetal, taxonomia, alimentação. Em uma aula sobre vitaminas e minerais, pode ser apresentada a riqueza nutricional presente nas PANC. A exemplo, temos a Amaranthus deflexus, espécie com alto teor nutritivo e potencial econômico (Kinupp; Lorenzi, 2017). Bessa (2011) fez uso de coleção herborizada em aulas práticas de morfologia vegetal, obtendo resultados satisfatórios.

O material destinado para leitura complementar possibilitará a retirada de possíveis dúvidas sobre as PANC e a coleção herborizada, além de complementar as informações contidas no manual. Ele pode ser facilmente encontrado na internet para download, com exceção de Kinupp e Lorenzi (2017), que está disponível apenas para compra. Com a popularização crescente da internet, a quantidade de informações presentes na rede é cada vez maior. A mesma é constituída por uma infinidade de páginas, nas quais é possível acessar informações de qualquer lugar e horário (Laruccia; Marcelino, 2008).

\section{Conclusão}

A coleção herborizada de PANC, além de apresentar a diversidade de plantas alimentícias, possibilita outras contribuições para a formação dos educandos. Entre elas, estão sensibilizar os alunos quanto à importância de espécies que são tidas como sem valor e utilidade tanto para o ser humano quanto para a natureza. Elas podem ser encontradas em suas casas, suas escolas, e em áreas públicas do estado de Sergipe.

Em relação ao manual da coleção, o mesmo pode auxiliar/ orientar o professor durante aulas de botânica. O professor irá elaborar aulas práticas de taxonomia, anatomia, morfologia vegetal, por exemplo, fazendo uso da coleção. Isto contribuirá para aulas mais participativas e atrativas, à medida que os alunos poderão observar e manusear as plantas e suas estruturas. Além disso, a coleção herborizada de PANC pode ir além das aulas de biologia. Os professores podem explorar o uso desse recurso da forma que considerarem mais apropriada, assim como dividi-lo com professores de outras áreas do ensino para a realização de um projeto em conjunto.

$\mathrm{Na}$ atualidade, é sabido que o ensino puramente tradicional não é suficiente para uma aprendizagem efetiva. A interação que ocorre na prática estimula a associação entre teoria e realidade. Portanto, faz-se necessário buscar alternativas para melhorar a qualidade do ensino e, consequentemente, formar cidadãos cientes do mundo que está a sua volta.

\section{Agradecimentos}

As autoras agradecem à equipe do Herbário da Universidade Federal de Sergipe (ASE) e do Laboratório de Sistemática Vegetal, pelo apoio; aos colegas que deram sugestões valiosas para a finalização deste trabalho; a M. V. Meiado, pela tradução da versão inicial do resumo em espanhol; a A. Mérnard e M. Dubena, pela tradução inicial do resumo em francês.

\section{Contribuições}

Conceitualização: ASF, MIUO. Curadoria de dados: ASF, MIUO. Análise formal: ASF, MIUO. Investigação: ASF. Metodologia: ASF, MIUO. Redação - rascunho original: ASF. Redação - revisão e edição: ASF, MIUO.

\section{Conflito de interesses}

As autoras declaram não haver conflitos de interesse a informar.

\section{Disponibilidade dos dados}

Os dados integrais analisados durante o estudo atual estão apresentados no corpo do manuscrito.

\section{Conformidade ética}

Não se aplica.

\section{Referências}

Acordi OSJ, Pasa MC. Trilha ecológica pedagógica como estratégia de ensino-aprendizagem nas escolas do município de Apiacás, MT, Brasil. Biodiversidade 2014;13(1):106-114.

APG IV. An update of the Angiosperm Phylogeny Group classification for the orders and families of flowering plants: APG IV. Botanical Journal of the Linnean Society 2016;181:1-20. doi: 10.1111/boj.12385

Barreira TF, Paula Filho GX, Rodrigues VCC, Andrade FMC, Santos RHS, Priore SE, Pinheiro-Sant'na HM. Diversidade e equitabilidade de Plantas Alimentícias Não Convencionais na zona rural de Viçosa, Minas Gerais, Brasil. Revista Brasileira de Plantas Medicinais 2015; 17(4):964-974. doi:10.1590/1983084X/14_100

Bessa MG. Montagem de coleção botânica para o auxílio do ensino de biologia no Ensino Médio. Monografia [Graduação em Ciências Biológicas] - Faculdade de Ciências da Educação e Saúde, Centro Universitário de Brasília; 2011.

Brasil. Base Nacional Comum Curricular (BNCC). Educação é a base. [Internet]. Ministério da Educação. 2018. [acesso em 4 jul 2020]. Disponível em: http://basenacionalcomum.mec.gov.br/

Brasil. Orientações Educacionais Complementares aos Parâmetros Curriculares Nacionais: Ciências da Natureza, Matemática e suas Tecnologias [Internet]. Ministério da Educação. 2006. [acesso em 20 mar 2018. Disponível em: http://portal.mec.gov.br/seb/ arquivos/pdf/CienciasNatureza.pdf

Carvalho IM (Org.). Didática: um estudo acadêmico. Campo Grande: UCDB; 2000.

Cordeiro J. Didática. São Paulo: Contexto; 2013.

Centro de Referência em Informação Ambiental - CRIA [Internet]. speciesLink. [acesso em 8 jan 2019]. Disponível em: http:// www.splink.org.br/index?lang=pt.

Esteves LM. Meio ambiente e botânica. São Paulo: Senac São Paulo; 2011.

Fagundes JA, Gonzalez CEF. Herbário escolar: suas contribuições ao estudo da botânica no Ensino Médio. 2006. [acesso em 20 set 2018]. Disponível em: http://www.diaadiaeducacao.pr.gov.br/ portals/pde/arquivos/1675-8.pdf

Ferreira JB, Libano AM. Confecção de um jogo didático para o ensino do bioma Cerrado: uma forma de sensibilização. Repositório Brasília: UniCEUB; 2011. [acesso em 23 fev 2019]. Disponível em: https://repositorio.uniceub.br/jspui/handle/235/6452 
Jardim Botânico do Rio de Janeiro [Internet]. Flora do Brasil 2020 em construção. [acesso em 10 mar 2019]. Disponível em: http:// floradobrasil.jbrj.gov.br/

Freire P. Pedagogia da autonomia: saberes necessários à prática educativa. Rio de Janeiro: Paz e Terra; 2013.

Gamarra-Rojas G, Gamarra-Rojas CFL, Barbosa MCA, Cruz LHV, Pereira SC. Jogo educativo: instrumento interativo na aprendizagem sobre plantas. In: Encontro Nacional de Biólogos, 5.; 2003 ago 3-6; Natal: CFBio; 2003.

[acesso em 20 set 2018]. Disponível em: http:// www.repositorio.ufc.br/handle/riufc/5388

Gattás MLB, Furegato ARF. A interdisciplinaridade na educação. Revista da Rede de Enfermagem do Nordeste 2017;8(1):85-91.

Kinupp VF. Plantas Alimentícias Não-Convencionais (PANCs): uma riqueza negligenciada. In: Reunião Anual da SBPC, 61.; 2009 jul 12-17; Manaus. Manaus: SBB; 2009. [acesso em 18 fev 2019]. Disponível em: https://grupos.moodle.ufsc.br/ file.php/346/referencias/PANCS-uma-riqueza-negligenciadaartigo-Kinupp.pdf

Kinupp VF, Barros IBI. Levantamento de dados e divulgação do potencial das plantas alimentícias alternativas no Brasil. Horticultura Brasileira 2004;22(2): CD-ROM.

Kinupp VF, Lorenzi H. Plantas Alimentícias Não Convencionais (PANC) no Brasil: guia de identificação, aspectos nutricionais e receitas ilustradas. São Paulo: Instituto Plantarum de Estudos da Flora; 2017.

Krasilchik M. Práticas de ensino de biologia. São Paulo: Edusp; 2016.

Laruccia MM, Marcelino SC. Ensaio sobre a informação e conhecimento na internet. In: Anais da $11^{\text {a }}$ SemeAd; 2008 ago 28-29, São Paulo. São Paulo: USP; 2008. [acesso em 23 fev 2019]. Disponível em: https://www.researchgate.net/ publication/242243937_Ensaio_sobre_a_Informacao_e_Conhecimento _na_Internet/link/566aa8a708ae430ab4f8004a/download

Maia LC, Vieira AOS, Peixoto AL, Canhos DA, Stehmann JR, Barbosa MRV, Menezes M. Construindo redes para promover o conhecimento da biodiversidade brasileira: a experiência do INCT - Herbário Virtual. Recife: UFPE; 2017.

Marandino M, Selles SE, Ferreira MS. Ensino de Biologia: histórias e práticas em diferentes espaços educativos. São Paulo: Cortez; 2009.

Menezes LC, Souza VC, Nicomedes MP, Silva NA, Quirino MR, Oliveira AG, Andrade RRD, Santos BAC. Iniciativas para o aprendizado de botânica no ensino médio. In: Encontro de iniciação à docência, 11.; 2008 abr 9-11; João Pessoa. João Pessoa: UFPB-PRG; 2008. [acesso em 15 ago 2018]. Disponível em: https://www.fernandosantiago.com.br/ensbot8.pdf

Mizukami MGN. Ensino: as abordagens do processo. São Paulo: EPU; 1986.

Ota MA. Herbário escolar: uma proposta de atividade prática para o ensino de Botânica. Monografia [Graduação em Ciências Biológicas] - Faculdade de Educação e Artes, Universidade Vale do Paraíba; 2012.

Peixoto AL, Maia LC (Orgs.) Manual de procedimentos para herbários. Recife: UFPE; 2013

Prata, APN, Amaral, MCE, Farias, MCV, Alves, MV Flora de Sergipe, volume 1. Aracaju: Triunfo; 2013

Santori RT, Santos MG (Orgs.) Ensino de ciências e biologia: um manual para a elaboração de coleções didáticas. Rio de Janeiro: Interciencia; 2015.

Santos MCF. Coleções biológicas para o ensino de ciências: o herbário didático do Instituto de Aplicação da UERJ. Cadernos do Aplicação 2013;6(1):11-18. doi: 10.22456/2595-4377.41179

Silva TS. A botânica na educação básica: concepções dos alunos de quatro escolas públicas estaduais em João Pessoa sobre o ensino de botânica. Monografia [Graduação em Ciências Biológicas] Universidade Federal da Paraíba; 2015.
Silva CM. Potencialidades do Cerrado: conhecer para proteger. Monografia [Graduação em Biologia a Distância] - Consórcio Setentrional de Educação a Distância, Universidade de Brasília, Universidade Estadual de Goiás; 2011.

\section{Una colección herborizada PANC como un re- curso didáctico para la enseñanza de la biología}

En la enseñanza de las Ciencias y Biología todavia persiste la memorización excesiva de conceptos y teorias, además de la descontextualización. Colecciones biológicas de las plantas alimenticias no convencionales (PANC) pueden ser una excelente manera de conectar el tema botánico con las plantas presentes en la vida cotidiana de los estudiantes. Así, se propuso como objetivo de este estudio, relatar la elaboración de una colección biológica didáctica de las PANC para ser utilizada por los maestros en las clases de Biología y contribuir con un aprendizaje más atractivo y significativo. A principio se buscó informaciones sobre las PANC y se seleccionaron especies presentes en el estado de Sergipe que podrian contribuir con el proceso de enseñanza y aprendizaje. Después de esta selección, se preparó un manual para la colección biológica. Debido a la baja motivación presente en la educación actual, se hace necesario buscar alternativas para mejorar la calidad de la educación y educar los ciudadanos conscientes del mundo que los rodea.

Palabras clave: Botánica. Ciencias. Colección biológica. Herbarios. Plantas alimenticias no convencionales.

\section{Une collection à base de plantes PANC comme resource pédagogique pour l'enseignement de la biologie}

Dans l'enseignement des sciences sociales et de la biologie, la mémorisation excessive des concepts et des théories persiste, outre leur décontextualisation. Lorsqu'elles sont liées aux plantes alimentaires non conventionnelles (PANC), les collections biologiques peuvent être un excellent moyen de connecter les connaissances botaniques avec les plantes présentes dans la vie quotidienne des élèves. C'est pourquoi ce travail visait à rendre compte l'élaboration d'une collection PANC à utiliser par les enseignants des cours de biologie, contribuant à un apprentissage plus attrayant et significatif. Des informations ont été recherchées sur les plantes $P A N C$ et les espèces sélectionnées présentes dans l'état de Sergipe qui pourraient contribuer au processus d'enseignement et d'apprentissage. Face à la faible motivation observée dans l'éducation actuelle, il est nécessaire de rechercher des alternatives pour améliorer la qualité de l'éducation et, par conséquent, d'éduquer les citoyens à prendre conscience du monde qui les entoure.

Mots clés: Botanique. Sciences. Collection biologique. Herbiers. Plantes alimentaires non conventionnelles. 